\title{
The fast rotating $\delta$ Scuti pulsator V376 Per: Frequency analysis and mode identification
}

\author{
Nathalie Themessl ${ }^{1}$, Veronique Fritz ${ }^{1,2}$, Michel Breger ${ }^{1,3}$, \\ Sabine Karrer ${ }^{1}$ and Barbara G. Castanheira ${ }^{3}$ \\ ${ }^{1}$ Institute for Astrophysics, University of Vienna, \\ Türkenschanzstraße 12, A-1180 Vienna, Austria \\ email: nathalie.themessl@univie.ac.at \\ ${ }^{2}$ Kapteyn Astronomical Institute, University of Groningen, The Netherlands \\ ${ }^{3}$ Department of Astronomy, University of Texas at Austin, USA
}

\begin{abstract}
Our simultaneous analysis of ground-based photometric and high-resolution spectroscopic data of the $\delta$ Scuti star V376 Per revealed eight individual frequencies from 82 nights of two-color photometry and six frequencies from the line-profile variations using 769 stellar spectra. Additionally, we identified the corresponding pulsation modes and derived reliable estimates of the line profile and pulsation mode parameters.
\end{abstract}

Keywords. stars: variables: $\delta$ Scuti, stars: interiors, line: profiles, methods: data analysis

\section{Introduction}

The $\delta$ Scuti star V376 Per (= HR 1170) was first detected to be variable by Breger (1969). The latest photometric and spectroscopic campaign $(2008-2011)$ provided more than 3800 hours of multi-color data obtained by the twin automatic photoelectric 75cm telescopes Wolfgang \& Amadeus at Fairborn Observatory in Arizona and 769 stellar spectra obtained with the 2.1-m Otto Struve telescope at McDonald Observatory in Texas, USA. While the multi-color measurements were corrected according to the threestar-technique (Breger 1993), we reduced the spectra with standard IRAF routines in order to remove effects caused by the instrument itself, the atmosphere and Earth's motion.

\section{Frequency analysis and mode identification}

In the first step, we derived the individual frequencies from the photometric lightcurves using Period04 (Lenz \& Breger 2004) and we extracted the spectroscopic frequencies in the pixels across the unblended Fe II line at $4508 \AA$ with FAMIAS (Zima 2008). Figure 1 illustrates both frequency spectra with the one-day aliases clearly visible in the spectral windows. The 12 most significant frequencies with an amplitude signal-to-noise ratio $(\mathrm{SNR}) \geqslant 4$ and the results of the subsequent mode identification are listed in Table 1. Due to the high rotational velocity of V376 Per we used the Fourier parameter fit method, embedded in FAMIAS, to determine the pulsation modes by means of the following stellar parameters: $M=1.71 M_{\odot}, R=2.39 R_{\odot}(\mathrm{Kim} \&$ Lee 1990$), T_{\text {eff }}=7100 \mathrm{~K}$, solar metallicity and $\log g=3.54$ (from Strömgren photometry).

\section{Discussion and conclusion}

The complementary photometric and spectroscopic studies provided useful constraints on the different modes of each detected frequency. In summary, the analysis provided 
Table 1. The most significant frequencies in the photometric $\left(f_{1}-f_{8}\right)$ and the spectroscopic $\left(\nu_{1}-\nu_{6}\right)$ dataset including the best solution for the mode identification.

\begin{tabular}{|c|c|c|c|c|c|}
\hline $\begin{array}{c}\text { Frequency } \\
{\left[\mathbf{d}^{-1}\right]}\end{array}$ & $\begin{array}{c}\text { A mplitude } \\
\text { in } v[\mathbf{m a g}]\end{array}$ & $\begin{array}{c}\text { Amplitude } \\
\text { in } y[\mathbf{m a g}]\end{array}$ & $\begin{array}{c}\text { Amplitude } \\
{\left[\mathbf{k m ~ s}^{-1}\right]}\end{array}$ & SNR & Mode identification \\
\hline$f_{1}=10.06$ & 0.0499 & 0.0346 & & 189 & $l=0$ \\
$f_{2}=11.91$ & 0.0240 & 0.0166 & & 91 & $l=1$ \\
$f_{3}=18.60$ & 0.0076 & 0.0053 & & 21 & $l=1$ \\
$f_{4}=9.69$ & 0.0049 & 0.0033 & & 18 & $l=0$ \\
$f_{5}=21.97$ & 0.0031 & 0.0022 & & 13 & $-1=0,1$ \\
$f_{6}=10.88$ & 0.0041 & 0.0029 & & 16 & $l=0,1$ \\
$f_{7}=19.71$ & 0.0022 & 0.0017 & & 7 & $l=1$ \\
$f_{8}=15.63$ & 0.0027 & 0.0019 & & 8 & $l=10, m=8$ \\
\hline$\nu_{1}=17.92$ & & & 1.176 & 13 & $l=3-4, m=2$ \\
$\nu_{2}=11.91$ & & & 0.710 & 11 & $l=6, m=1,2,3,4$ \\
$\nu_{3}=14.10$ & & & 0.622 & 9 & $l=9, m=3,5,7,9$ \\
$\nu_{4}=16.97$ & & & 0.594 & 7 & $l=0, m=0$ \\
$\nu_{5}=10.06$ & & & 0.447 & 11 & $l=0,5,6$ \\
$\nu_{6}=23.67$ & & & 5 & $l=10-11, m=4,5,6$ \\
\hline
\end{tabular}
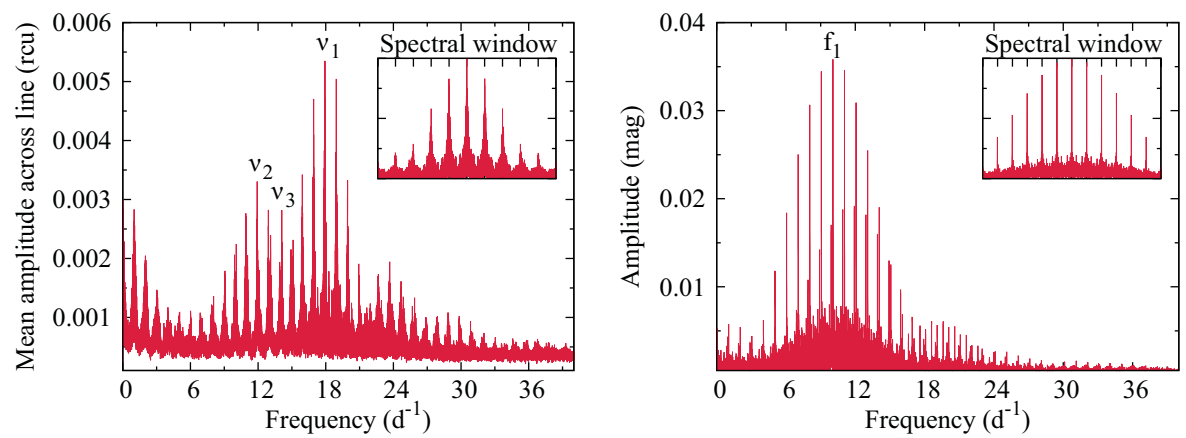

Figure 1. Left: The one-dimensional Fourier spectrum of the original spectroscopic dataset revealing the first three frequencies with the highest amplitude (in relative continuum units). Right: The general frequency spectrum of the combined photometric datasets ( $y$ and $v$ ) with the highest peak at $10.06 \mathrm{~d}^{-1}$. The one-day alias peaks are clearly visible in both spectral windows.

low $l$-values for the photometrically detected frequencies and $l>5$ for those detected in the spectroscopic dataset. We note that the line-profile analysis is capable of detecting (and also favoring) high-degree modes with an uncertainty of \pm 1 in $l$. For this reason, we attained a slightly higher degree for $\nu_{2}=11.91 \mathrm{~d}^{-1}$ by applying the spectroscopic method compared to the more reliable photometric determination of the same frequency. Moreover, the projected equatorial rotational velocity $(v \sin i)$ could be constrained to $100 \pm 2 \mathrm{~km} \mathrm{~s}^{-1}$ suggesting a favored inclination between 35 and 75 degrees. Additionally, the computation of different evolution models revealed $f_{1}$ as the radial first overtone, which in turn specifies the evolutionary state of V396 Per. The evolutionary track of the model also suggests that the star no longer resides on the main sequence.

\section{References}

Breger, M. 1969, AJ, 74, 166

Breger, M. 1993, Proc. of the IAU Colloquium, 136, 106

Kim, S. \& Lee, S. 1990, Journal of the Korean Astron. Society, 23, 1

Lenz, P. \& Breger, M. 2004, CoAst, 146, 53

Zima, W. 2008, CoAst, 155, 17 